\title{
Mechanism of the JAK2/STAT3-CAV-1-NR2B signaling pathway in painful diabetic neuropathy
}

\author{
Chuan-Da Li ${ }^{1} \cdot$ Jia-Yi Zhao ${ }^{1} \cdot$ Jia-Li Chen ${ }^{1} \cdot$ Jia-Hui Lu ${ }^{1}$ Mao-Biao Zhang ${ }^{1} \cdot$ Qi Huang ${ }^{1} \cdot$ Yan-Nan Cao ${ }^{1} \cdot$ Gai-Li Jia $^{1}$. \\ Yuan-Xiang $\mathrm{TaO}^{2} \cdot$ Jun $\mathrm{Li}^{1} \cdot \mathrm{Hong} \mathrm{Cao}^{1}$
}

Received: 24 October 2018 / Accepted: 22 February 2019 / Published online: 4 March 2019

(C) The Author(s) 2019

\begin{abstract}
Purpose The aim of the present study was to further elucidate the role of JAK2/STAT3-CAV-1-NR2B on painful diabetic neuropathy.

Methods In vivo, the mechanical withdrawal threshold and thermal withdrawal latency were measured to evaluate neuropathic pain behaviors $(n=8)$, while western blot $(n=5)$ and an immunofluorescence double staining experiment $(n=6)$ were performed to understand the molecular mechanism. In vitro, the individual culture of BV2 mouse microglia cell lines, the co-culture of BV2 mouse microglia cell lines and PC12 rat neuron cell lines, and western blot analysis were performed to understand the molecular mechanism between microglia and neurons.

Results The expression of p-JAK2, p-STAT3, t-CAV-1, and p-NR2B was upregulated in the dorsal horn of DNP rats throughout the experiment. Through the immunofluorescence double staining experiment, it was found that p-STAT3 was mainly expressed in activated microglia, and this condition can be stably maintained for approximately 2 weeks after the establishment of the DNP model. The intrathecal injection of JAK2 inhibitor AG490 can relieve the abnormal expression of p-JAK2, p-STAT3, t-CAV-1, and p-NR2B, and relieve pain. The remission of AG490 began on the third day, and it could be stably sustained for 14 days. In vitro high-glucose induced the activation of p-STAT3 in microglia, thereby upregulating the expression of p-CAV-1 and p-NR2B in neurons in the co-culture system. JAK2 inhibitor AG490 can alleviate the abnormal expression of these proteins in the JAK2/STAT3-CAV-1-NR2B signaling pathway in vitro.
\end{abstract}

Conclusions Microglial JAK2/STAT3 signaling probably contributes to neuropathic pain by activating the CAV-1-NR2B pathway.

Keywords Painful diabetic neuropathy $\cdot \mathrm{JAK} 2 / \mathrm{STAT} 3 \cdot \mathrm{CAV}-1 \cdot \mathrm{NR} 2 \mathrm{~B}$

\section{Abbreviations}

T2DM Type-2 diabetic mellitus

DNP Painful diabetic neuropathy

NMDA N-methyl-D-aspartate receptor

DRG Dorsal root ganglion

Jun Li

lijun0068@163.com

$\triangle$ Hong Cao

caohong1955@21cn.com

1 Department of Anesthesiology, Second Affiliated Hospital of Wenzhou Medical University, Pain Medicine Institute of Wenzhou Medical University, 325035 Zhejiang, China

2 Department of Anesthesiology, New Jersey Medical School, Rutgers, The State University of New Jersey, Newark, NJ 07103, USA
JAK2 Janus Kinase 2

STAT3 Signal transducers and activators of transcription

CAV-1 Caveolin-1

NR2B NMDA receptor 2B

HFSD High-fat-sugar diet

STZ Streptozocin

MWT Mechanical withdrawal threshold

TWL Thermal withdrawal latency

DMSO Dimethylsulfoxide

AG-490 Tyrphostin AG490

CCI Chronic constrictive injury

ISI Insulin sensitivity index

BDNF Brain-derived neurotrophic factor 


\section{Introduction}

Type-2 diabetic mellitus (T2DM) has reached a pandemic status, and has shown no signs of abatement. Painful diabetic neuropathy (DNP) has been generally considered to be one of the most common complications of T2DM with a $30-50 \%$ incidence, and it has also been recognized as one of the most difficult types of pain to treat [1]. Glycemic control and the use of analgesic drugs are the primary treatments for DNP. However, these are accompanied by unobvious curative effects. Exploring the pathogenesis of DNP would be helpful for developing novel therapeutic strategies.

Spinal cord dorsal horn glial cell signal transductioninduced central sensitization is one of the important pathogeneses of DNPs. The activation of microglia produces and releases a variety of cytokines and excitatory substances of neurons or glial cells [2-4], promotes NR2B activation on neuronal cells, NR2B is a subunit on NMDA, and increases NMDA-mediated current. NMDA receptor activation can further induce the sensitization of the central nervous system, which is one of the important pathogeneses of DNP $[5,6]$.

JAK2/STAT3 signal transduction, as a classical intracellular signal transduction pathway, participates in many pathophysiological processes, such as cell differentiation, proliferation, inflammation, and pain formation. The activation of microglia caused by JAK2/STAT3 has a significant effect on neuropathic pain. After nerve injury induces vigorous IL-6 production in dorsal root ganglion (DRG), the cytokines may be transported to central terminals of primary afferents. The released cytokines stimulate the JAK/STAT3 signaling pathway in spinal microglia and promote genesis of neuropathic pain. Meanwhile, these cytokines further activate glial cells and neurons to release more activating substances such as ATP, pro-inflammatory factors, reactive oxygen species (ROS), nitric oxide (NO), prostaglandins (PGs), etc. These activating substances further enhance neuropathic pain [7]. In addition, JAK2/ STAT3 signal transduction has an effect on spinal NMDAinduced currents, causing neuropathic pain [8]. However, the relationship between the JAK2/STAT3 pathway and activation of microglia in DNP remains unclear.

Caveolae are a specialized type of lipid raft that are stabilized by oligomers of the caveolin protein. Caveolin-1 (CAV-1), a major protein component of caveolae, is an important gene targeting for STAT3. The overexpression of p-STAT3 can cause CAV-1 promoter activation and increase gene expression [9]. CAV-1 regulates neuronal plasticity and receptor transport to regulate NR2BNMDAR, which is closely correlated with pathologic pain and central sensitization [10].
However, no studies on DNP have focused on the JAK2/ STAT3 signaling pathway. Therefore, the present study further explored the correlation between DNP and microglial activation by combining in vivo and in vitro experiments to explore the role of this signaling pathway in the pathogenesis of DNP, and explore its regulation of the downstream of CAV-1 and NR2B.

\section{Materials and methods}

\section{Animals}

The study protocol was approved by the Animal Research Committee of Wenzhou Medical University. All animal experiments were performed in accordance with the National Institutes of Health Guidelines for the Care and Use of Laboratory Animals, and in accordance with the Animal Research: Reporting In Vivo Experiments (ARRIVE) guidelines. Sprague-Dawley (SD) rats, weighing $120-160 \mathrm{~g}$, were provided by the Center for Laboratory Animals of Wenzhou Medical University (License No. WYDW2014-0015). These rats were housed in room temperature, which was maintained within $23-25^{\circ} \mathrm{C}$, allowed free access to food and water, and placed under a 12-hour/ 12-hour dark/light cycle. The detailed design schemes were depicted in Figs 1 and 2.

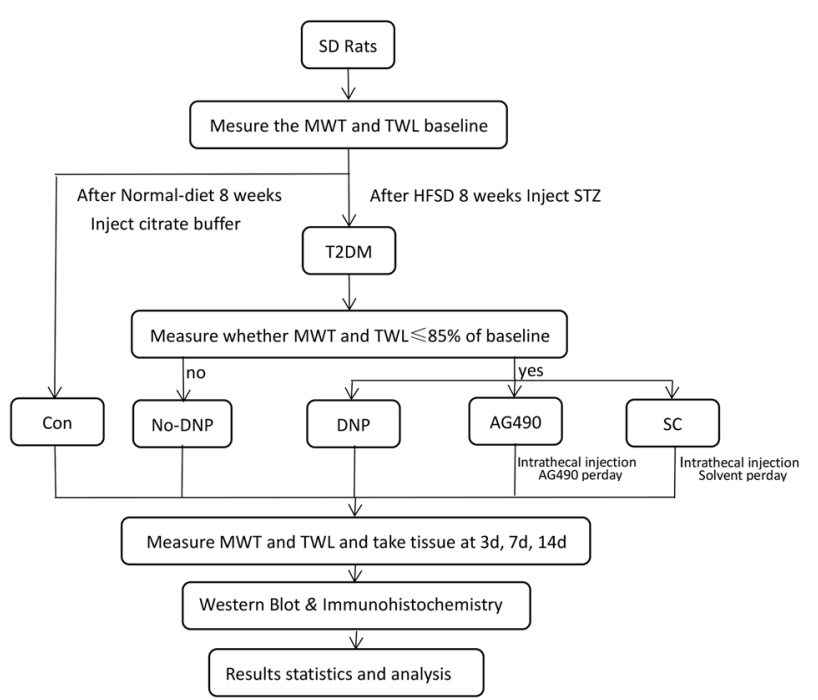

Fig. 1 In vivo experiment design scheme. After 3 days of injection, conditions with blood glucose levels $\geqq 16.7 \mathrm{mmol} / \mathrm{L}$ were considered as T2DM. Rats in the AG490 group and dimethylsulfoxide (DMSO) group were injected, respectively, with $10 \mu \mathrm{L}(1 \mathrm{mmol} / \mathrm{L})$ of AG490 and $10 \mu \mathrm{L}$ of $3.5 \%$ DMSO once a day for 14 days. Mechanical withdrawal threshold (MWT) and thermal withdrawal latency (TWL) were measured before streptozocin (STZ) injection, on day 3 after STZ injection (as a reference for successful modeling) and on days 3,7 , and 14 after intrathecal injection $(n=5)$ 


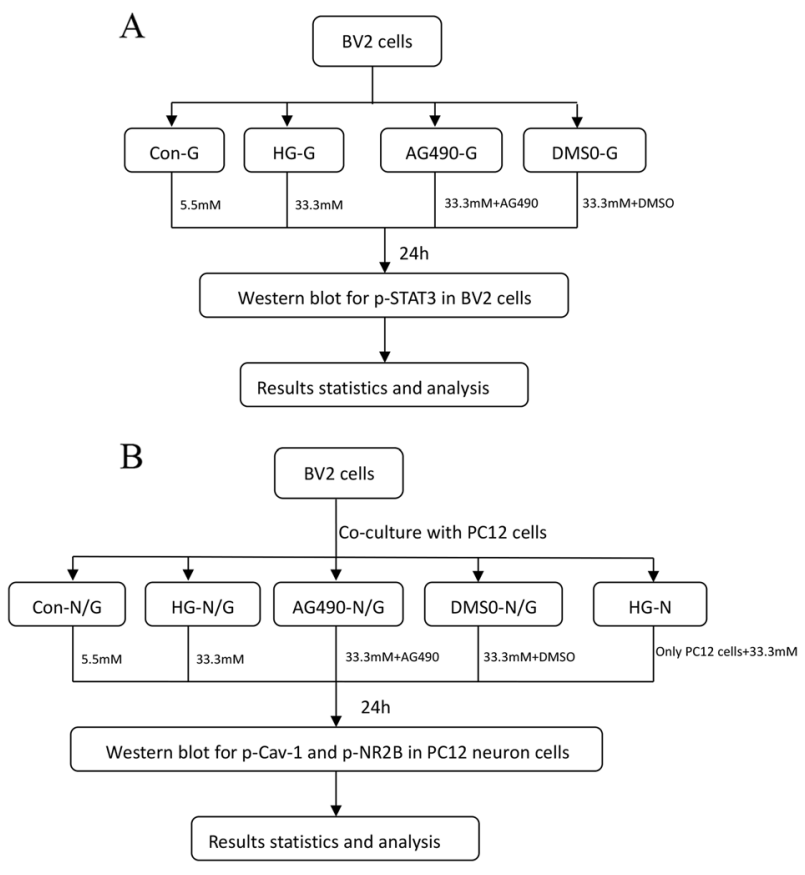

Fig. 2 In vitro experiment design scheme. A Step 1 BV2 cells were seeded at $1 \times 10^{6}$ cells/well in a 6-well plate. Con-G: BV2 cells treated with low glucose (5.5 mM D-glucose); HG-G: BV2 cells treated with high glucose (33.3 mM D-glucose); AG490-G: BV2 cells treated with AG490 $(50 \mu \mathrm{M})+$ high glucose; DMSO-G: BV2 cells treated with dimethylsulfoxide (DMSO) + high glucose. After $24 \mathrm{~h}$ of culture, western blot was used to detect the expression of p-STAT3 in BV2 microglia cells. B Step 2 Transwell was used to establish a co-culture system of BV2 cells and PC12 cells. Con-N/G: BV2 cells and PC12 cells treated with low glucose $(5.5 \mathrm{mM}$ D-glucose); HG- N/G: BV2 cells PC12 cells treated with high glucose $(33.3 \mathrm{mM}$ D-glucose); AG490- N/G: BV2 cells PC12 cells treated with AG490 $(50 \mu \mathrm{M})+$ high glucose; DMSO-N/G: BV2 cells PC12 cells treated with DMSO + high glucose. HG-N: PC12 cells treated with low glucose. After $24 \mathrm{~h}$ of culture, western blot was used to detect the expression of p-Cav-1 and $\mathrm{p}-\mathrm{NR} 2 \mathrm{~B}$ in $\mathrm{PC} 12$ cells

\section{Induction of T2DM and DNP}

These T2DM models were established, as previously described [11, 12]. After 2 weeks of adaptive feeding, these rats were randomized into two groups: Con group and T2DM group. Rats in the T2DM group were fed with a high-fat-sugar diet (HFSD) for 8 weeks, while rats in the Con group were fed with a normal-diet. After 8 weeks, the insulin sensitivity trail was performed by testing for fasting blood glucose and insulin on blood samples collected from the tail vein. Insulin sensitivity index (ISI) $=1 /$ (blood glucose $\times$ insulin). These results revealed that there was a significant difference in ISI between these two groups $(P<$ $0.05)$, implying that insulin resistance was successfully induced in the T2DM group. Subsequently, rats in the T2DM group were intraperitoneally injected with streptozocin (STZ) (Sigma Co., St. Louis, MO, USA) at a dose of
$35 \mathrm{mg} / \mathrm{kg}$. As a control, rats in the Con group were intraperitoneally injected with the same dose of citrate buffer. After three days of injection, rats in the T2DM group had a caudal vein fasting blood glucose of $\geq 16.7 \mathrm{mmol} / \mathrm{L}$, and were considered as T2DM rats. After measuring the mechanical withdrawal threshold (MWT) and thermal withdrawal latency (TWL) of these rats, rats with MWT and TWL values $\leq 85 \%$ of baseline (before the high-fat-sugar diet) were considered as a successful model of type-2 painful diabetic neuropathy, and was redefined as the TDNP group. The remaining rats in the T2DM group were redefined as the No-DNP group.

\section{Subarachnoid catheterization and AG490 administration}

AG490, a specific inhibitor of JAK2, inhibits the activation of STAT3 by selectively blocking JAK2. AG490 (1 mmol/L) was dissolved in 3.5\% dimethylsulfoxide (DMSO), and 3.5\% DMSO was used as the control vehicle (DMSO group). Rats in the T-DNP group were randomly divided into three groups: DNP group, AG490 group, and DMSO group. Rats in the AG490 and DMSO groups received subarachnoid catheterization, as previously described. Under anesthesia with chloral hydrate $(0.3 \mathrm{~g} / \mathrm{kg}$ ), a PE-10 silastic tubing (Ningbo Science and Technology Park to the Software Technology Co., Ltd, China) was intrathecally inserted between the L4 and L5 vertebrae, and advanced $2 \mathrm{~cm}$ into the lumbar enlargement of the spinal cord. The external end of the intrathecal catheter was tunneled under the skin to the neck area, and the outer part of the catheter was exposed, carefully plugged and fixed onto the skin. The animals were allowed to recover for 5-6 days after the surgery. In order to verify the location of the catheter, $10 \mu \mathrm{L}$ of $2 \%$ lidocaine was given through the catheter. Limb paralysis response without neurological deficits indicated that the insertion was successful. Then, the rats in the AG490 group and DMSO group were injected, respectively, with AG490 $10 \mu \mathrm{L}(1 \mathrm{mmol} / \mathrm{L})$ and $3.5 \%$ DMSO $10 \mu \mathrm{L}$ once a day for 14 days.

\section{Behavioral tests}

MWT and TWL were measured before STZ injection, on day 3 after STZ injection (as a reference for successful modeling) and on days 3, 7, and 14 after intrathecal injection. The detained behavioral tests and model preparation were described in the Supplementary Methods.

\section{Cell cultures and treatments}

BV2 immortalized murine microglia cell line was purchased from MX Biotechnology Company (Shanghai, China). PC12 neuronal cell line cells were provided by the College 
of Pharmacy of Wenzhou Medical University. The cells were cultured in Dulbecco's modified eagle's medium (DMEM) supplemented with $10 \%$ fetal bovine serum (FBS), $100 \mathrm{U} / \mathrm{mL}$ of penicillin, $100 \mu \mathrm{g} / \mathrm{mL}$ of streptomycin, and $5.5 \mathrm{mmol} / \mathrm{L}$ of glucose.

\section{BV2 microglia cell line cultures and treatments}

BV2 cells were seeded at $1 \times 10^{6}$ cells/well in a 6-well plate. BV2 microglia cells were randomly divided into four groups: control group (Con-G group), high-glucose group (HG-G group), JAK2 inhibitor group (AG490-G group), and solvent control group (DMSO-G group). BV2 cells were cultured with low-glucose medium $(5.5 \mathrm{mM}$ D-glucose) to the exponential phase. Then, BV2 cells were replaced with low-glucose medium (5.5 mM D-glucose), high-glucose medium (33.3 mM D-glucose), AG490 $(50 \mu \mathrm{M})$ plus high-glucose medium (33.3 $\mathrm{mM}$ D-glucose), and DMS plus high-glucose medium (33.3 mM D-glucose), respectively. After $24 \mathrm{~h}$ of culture, western blot was performed to detect the expression of p-STAT3 in BV2 microglia cells.

\section{BV2 microglia cell co-culture with PC12 neuron cells}

Transwell was used to build the co-culture system of BV2 microglia cells and PC12 neuron cells. These cells were randomly divided into five groups: control group (Con-N/G group), high-glucose group (HG-N/G group), JAK2 inhibitor group (AG490-N/G group), solvent control group (DMSO-N/G group), and neuron high-glucose group (HG$\mathrm{N}$ group). In the HG-N group, $\mathrm{PC} 12$ cells were seeded in a six-well plate with no cells seeded in the transwell, which was inside the six-well plate. For the other four groups, PC12 cells were seeded in a six-well plate, and BV2 cells were seeded in the transwell, which was inside the six-well plate. Cells in the Con-N/G group, HG-N/G group, AG490N/G group, DMSO-N/G group, and AG490-N/G group were cultured with low-glucose medium $(5.5 \mathrm{mM}$ D-glucose) to the exponential phase, and this was subsequently replaced with low-glucose medium (5.5 mM D-glucose), high-glucose medium (33.3 mM D-glucose), AG490 $(50 \mu \mathrm{M})$ plus high-glucose medium (33.3 $\mathrm{mM}$ D-glucose), DMSO plus high-glucose medium (33.3 mM D-glucose), and high glucose (33.3 mM D-glucose), respectively. After $24 \mathrm{~h}$, western blot analysis was carried out to detect the expression of p-CAV-1 and p-NR2B in PC12 cells.

\section{Western blot analysis}

Protein samples were separated using $10 \%$ sodium dodecyl sulfate polyacrylamide gel electrophoresis (SDS-PAGE, $60 \mathrm{~g}$ of total protein per lane) and transferred onto a polyvinylidene fluoride membrane (Merck Millipore, Temecula, CA, USA). In addition, gels stained with Coomassie Blue were used to confirm the equal amounts of protein loaded on each lane. The membranes were incubated overnight at $4{ }^{\circ} \mathrm{C}$ with primary polyclonal rabbit antip-CAV-1 (1:500; Santa Cruz Biotechnology, Santa Cruz, CA, USA), anti-t-CAV-1 antibody (1:500; Santa Cruz Biotechnology, Santa Cruz, CA, USA), anti-p-JAK2 (1:500; Abcam, Cambridge, MA, USA), anti-p-STAT3 (1:1000; Cell Signaling Technology, Danvers, MA, USA), or anti-p-NR2B antibody (1:500; Millipore, Billerica, MA, USA). The membranes were extensively applied with Tris buffered saline Tween 20 (TBST) and incubated for $2 \mathrm{~h}$ with horseradish peroxidase conjugated secondary antibody (1:3000; Abcam, UK)) at room temperature. The immune complexes were detected using a nitro blue tetrazolium $/ 5$ bromo-4-chloro-3-indolyl phosphate assay kit (Sigma Co., St. Louis, MO, USA), or chemiluminescence (Pierce, Waltham, MA, USA). The density of the specific bands was analyzed using NIH ImageJ software, and the expression levels of these proteins were normalized to $\beta$-actin.

\section{Immunohistochemistry}

Rats were deeply anaesthetized with $400 \mathrm{mg} / \mathrm{kg}$ of $5 \%$ chloral hydrate, transcardially perfused with $200 \mathrm{~mL}$ of phosphate buffered saline (PBS, $0.01 \mathrm{M}$; Solarbio Science \& Technology, Beijing, China), followed by $300 \mathrm{~mL}$ of freshly prepared $4 \%$ paraformaldehyde in $0.01 \mathrm{M}$ PBS. The fourth to sixth lumbar segments of the spinal cord were removed, post-fixed with the same fixative for $12 \mathrm{~h}$ at $4{ }^{\circ} \mathrm{C}$, and placed in $10 \%, 20$ and $30 \%$ (w/v) sucrose solutions, one by one, for $12 \mathrm{~h}$ at $4{ }^{\circ} \mathrm{C}$. Then, the dehydration spinal cord tissue was embedded with OTC, $20 \mu \mathrm{m}$ was cut by a frozen section machine at a constant temperature of $-20^{\circ} \mathrm{C}$, washed with $0.01 \mathrm{M}$ PBS solution at $4{ }^{\circ} \mathrm{C}$ three times for $20 \mathrm{~min}$ each time, and incubated in a blocking solution ( $0.2 \%$ Trition; $3 \%$ goat serum; $0.01 \mathrm{M}$ PBS) for $1 \mathrm{~h}$ at $26^{\circ} \mathrm{C}$. Then, the tissue was incubated for $24 \mathrm{~h}$ at $4{ }^{\circ} \mathrm{C}$ with the following primary antibodies: p-STAT3 (1:200; Pierce, Waltham, MA, USA), GFAP (1:1000; Merck Millipore, Temecula, CA, USA), OX-42 (1:200; Abdserotec, Hercules, CA, USA), and Neu (1:800; Merck Millipore, Temecula, CA, USA). After incubation, the sections were placed in room temperature for $1 \mathrm{~h}$, washed three times ( $10 \mathrm{~min}$ each time) with $0.01 \mathrm{M}$ PBS solution at $4{ }^{\circ} \mathrm{C}$, and incubated for $2 \mathrm{~h}$ at $26^{\circ} \mathrm{C}$ with the following secondary antibodies: Alexa Fluor ${ }^{\circledR} 546$ Goat Anti-Mouse IgG, and Alexa Fluor ${ }^{\circledR} 488$ Goat Anti-Rabbit IgG (1:1,000; Invitrogen, Waltham, MA USA). Then, the tissues were washed with $0.01 \mathrm{M}$ PBS solution at $4{ }^{\circ} \mathrm{C}$ three times at $20 \mathrm{~min}$ each time. The slices were observed by fluorescence microscopy and analyzed by Image-Pro Plus software. 
Table 1 Comparison of blood glucose, insulin level, and insulin sensitivity index (ISI) after normal-diet (Con) or a high-fat-sugar diet (T2DM) $($ Mean $\pm \mathrm{SD}, n=8)$

\begin{tabular}{|c|c|c|c|c|c|c|}
\hline \multirow[t]{2}{*}{ Group } & \multicolumn{2}{|c|}{ Blood glucose levels (mmol/L) } & \multicolumn{2}{|c|}{ Insulin levels (mIU/L) } & \multicolumn{2}{|l|}{ ISI } \\
\hline & Baseline & $\begin{array}{l}\text { After high-fat-sugar diet } \\
\text { (HFSD) }\end{array}$ & Baseline & After HFSD & Baseline & After HFSD \\
\hline Con & $2.89 \pm 0.47$ & $4.60 \pm 0.54$ & $7.51 \pm 2.00$ & $9.36 \pm 2.87$ & $-3.22 \pm 0.41$ & $-3.15 \pm 0.43$ \\
\hline $\mathrm{T} 2 \mathrm{DM}$ & $3.65 \pm 1.23$ & $5.10 \pm 0.90$ & $8.01 \pm 2.44$ & $27.78 \pm 6.09^{*}$ & $-3.08 \pm 0.56$ & $-4.51 \pm 0.39^{*}$ \\
\hline
\end{tabular}

${ }^{*} P<0.05$ vs. Con group

\section{Data analysis}

Data were presented as mean \pm standard deviation (SD). The results were statistically analyzed using one-way analysis of variance (ANOVA), or paired or unpaired Student's $t$-test. When the ANOVA results revealed a significant difference, pairwise comparisons between means were tested by the least significant difference method (LSD). These data were analyzed by SPSS 19.0 (SPSS Inc., Chicago, IL, USA). $P<0.05$ was considered statistically significant.

\section{Results}

\section{Changes in blood glucose level, insulin level, and insulin sensitivity index}

After 8 weeks of HFSD, insulin resistance was successfully elicited. Compared with the Con group, rats in the T2DM group had a higher blood glucose level $(P<0.05)$. However, this was not high enough to meet the diagnostic criteria of T2DM ( $>16.7 \mathrm{mmol} / \mathrm{L}$ ), although rats in the T2DM group had a higher insulin level and lower insulin sensitivity index $(P<0.05$, Table 1$)$. At three days after STZ injection, the levels of blood glucose in diabetic rats dramatically increased, when compared with control rats, and this high blood glucose level was maintained until the end of the experiments $(P<0.05$, Fig. 3$)$. The average levels in the diabetic rats were higher than $16.7 \mathrm{mmol} / \mathrm{L}$, indicating that the type-2 diabetic models were successful established.

\section{MWT and TWL}

Compared with the Con group, the MWT and TWL of the DNP and DMSO groups both significantly decreased $(P<$ 0.05). Compared with DNP group, the MWT and TWL of the AG490 group increased after AG490 intrathecal administration $(P<0.05)$. The reductions in MWT and TWL were time-dependently reversed in type- 2 DNP rats after the administration of AG490. These results indicate that the injection of AG490 alleviated the mechanical hyperalgesia and thermal allodynia in type-2 DNP rats. Furthermore, no significance difference was found between the Con and no-DNP groups (Fig. 4).

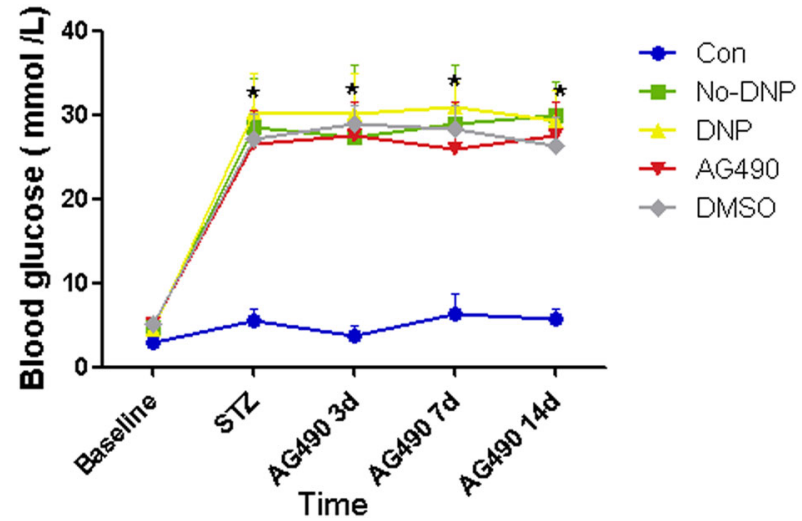

Fig. 3 Comparison of blood glucose between groups (Mean $\pm \mathrm{SD}, n=$ 8 ). The comparison of blood glucose before and at 3 days after administration of streptozocin (STZ) and subarachnoid administration at $3,7,14$ days $(n=8),{ }^{*} P<0.05$ vs. Con group

\section{The JAK2/STAT3-CAV-1-NR2B signal pathway was activated in the spinal dorsal horn of DNP and could be inhibited by AG490 in vivo}

Compared with the Con group, the levels of p-JAK2, pSTAT3, t-CAV-1, and p-NR2B in the DNP group significantly increased $(P<0.05)$. As shown in Fig. 5, the administration of AG490 blocked the increase in p-JAK2, p-STAT3, t-CAV-1, and p-NR2B levels in the spinal dorsal horn of type-2 DNP rats $(P<0.05)$. However, there was no significant difference between the DNP and DMSO groups at any time point $(P>0.05$, Fig. 5).

\section{The p-STAT3 mainly co-labeled with microglial cells in the spinal cord of DNP in vivo}

In order to examine which types of cells in the dorsal horn of type-2 DNP rats expressed p-STAT3, double-labeled immunofluorescent staining was carried out. The result revealed that p-STAT3 accumulated mainly in the superficial and medial laminae (I-V) of the spinal cord horn, and more p-STAT3-positive cells were found in the DNP group (Fig. 6Aa-b, Ba-b, Ca-b). Immunofluorescence double staining experiments were performed to identify the cell types. Compared with astrocytes and neurons, p-STAT3-IR mainly coexisted with the microglia (Fig. 6Ac-h, Bc-h, Cc-h). 

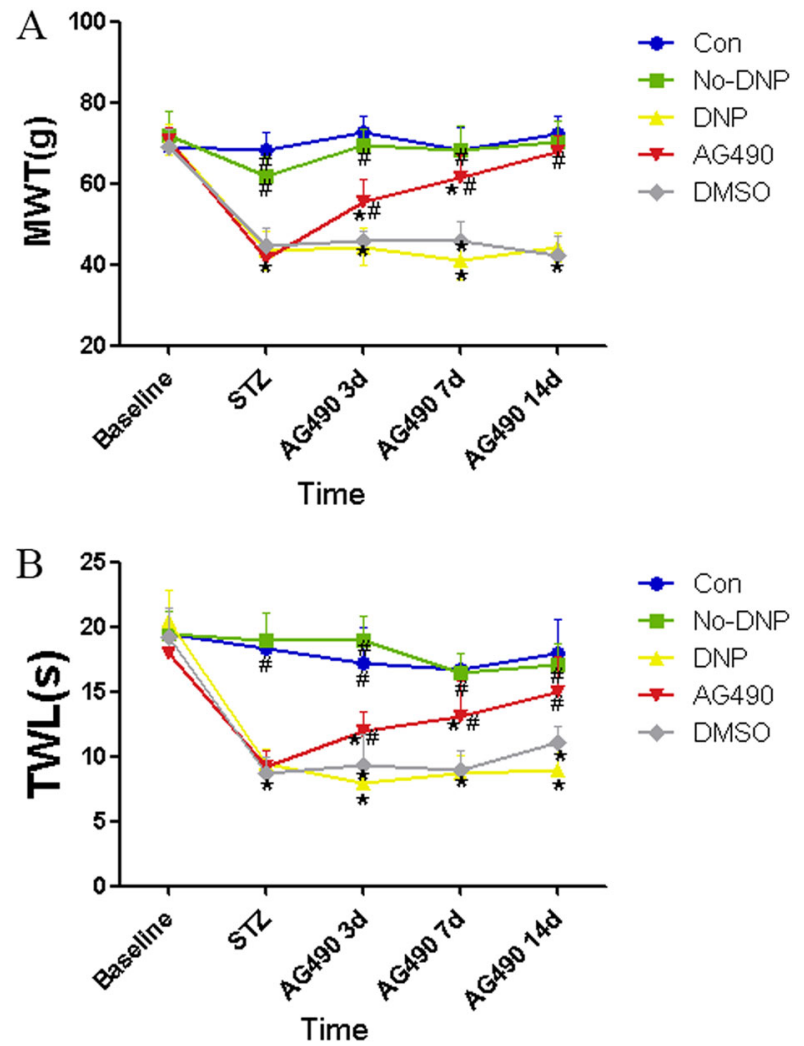

Fig. 4 Comparison of mechanical withdrawal threshold (MWT) and thermal withdrawal latency (TWL) between groups. A The comparison of MWT before and at 14 days after administration of streptozocin (STZ) and subarachnoid administration at 3, 7, 14 days $(n=8)$. $* P<0.05$ vs. Con group, $\# P<0.05$ vs. painful diabetic neuropathy (DNP) group. B The comparison of TWL before and at 14 days after administration of STZ and subarachnoid administration at 3, 7, 14 days $(n=8) . * P<0.05$ vs. Con group, $\# P<0.05$ vs. DNP group

\section{High-glucose induced the activation of p-STAT3 in BV2 cells and upregulated the expression of p-CAV-}

\section{1 and p-NR2B in PC12 cells, which could be inhibited by $A G 490$ in vitro}

The results of the BV2 cell culture revealed that the expression of p-STAT3 in BV2 cells was upregulated in the HG-G group, when compared with the Con-G group $(P<$ 0.001 ). Moreover, compared with the HG-G group, the expression of p-STAT3 was downregulated in the AG490$\mathrm{G}$ group $(P<0.01)$. However, there was no significant difference between the HG-G and DMSO-G groups. The results of the co-culture system revealed that the expression levels of p-CAV-1 and p-NR2B in PC12 cells were upregulated in the HG-N/G group, when compared with the Con-N/G and HG-N groups $(P<0.01)$. However, when compared with the HG-N/G group, the expression levels of p-CAV-1 and p-NR2B were downregulated in the AG490-
N/G group $(P<0.01)$, and there was no significant difference between the HG-N/G and DMSO-N/G groups (Fig. 7).

\section{Discussion}

In the present study, diabetic rats were induced not only by STZ injection, but also by combining with HFSD feeding, in order to elicit insulin resistance, which would better reflect the pathophysiological process of T2DM. Moreover, the subsequent induced DNP rat model could steadily be maintained for approximately 2 weeks both in pain threshold and blood glucose.

\section{JAK2/STAT3 and the activation of microglia}

JAK2/STAT3 signal transduction is involved in the activation of glial cells and formation of neuropathic pain [13]. In the CCI model, the presence of neuropathic pain was accompanied by the high expression of JAK2 and STAT3, and the inhibition of the expression of these factors could significantly relieve pain [14]. Ion channel dysfunction is central to the pathogenesis of painful DNP. In hippocampal neurons, the JAK/STAT signaling pathway is closely correlated to NMDAR function. IL-6 regulates the $\mathrm{Ca}^{2+}$ influx of NMDAR through the JAKs/STATs signal pathway, resulting in neurodegenerative lesions [15]. The activation of JAK2 further activates STAT3, and activated stat 3 forms a dimer, which is involved in the regulation of the transcription of inflammatory factor genes, possibly acting on neuronal NMDARs, and causing central sensitization, leading to DNP. In the present study, it was found that JAK2 activity increased in DNP rats, while its inhibitor AG490 could downregulate p-JAK2 and p-STAT3 in vivo and in vitro, and relieve pain.

Signal transducers and activators of transcription (STAT) is a unique protein that can induce the expression of target genes [16]. In recent years, studies have found that the activation of STAT3 subtypes is involved in the transmission and modulation of nociceptive information in spinal cord levels after peripheral injury [7]. In the SCI model, and early in the spinal nerve injury, the activation of the JAK2/ STAT3 pathway was found in activated microglia [17].

The microglia and astrocytes were both considered to be involved in neuropathic pain, and a researcher supposed that microglia participates in the early phase of pain, while astrocytes contribute to the later phase. The activation of microglia can transmit the abnormal pain signal to neurons of the spinal cord, thereby contributing to the development of neuropathic pain [18]. In spinal nerve ligation-induced pain and bone cancer-induced pain, the activation of microglia is present, and the spinal inhibition of microglia by minocycline 

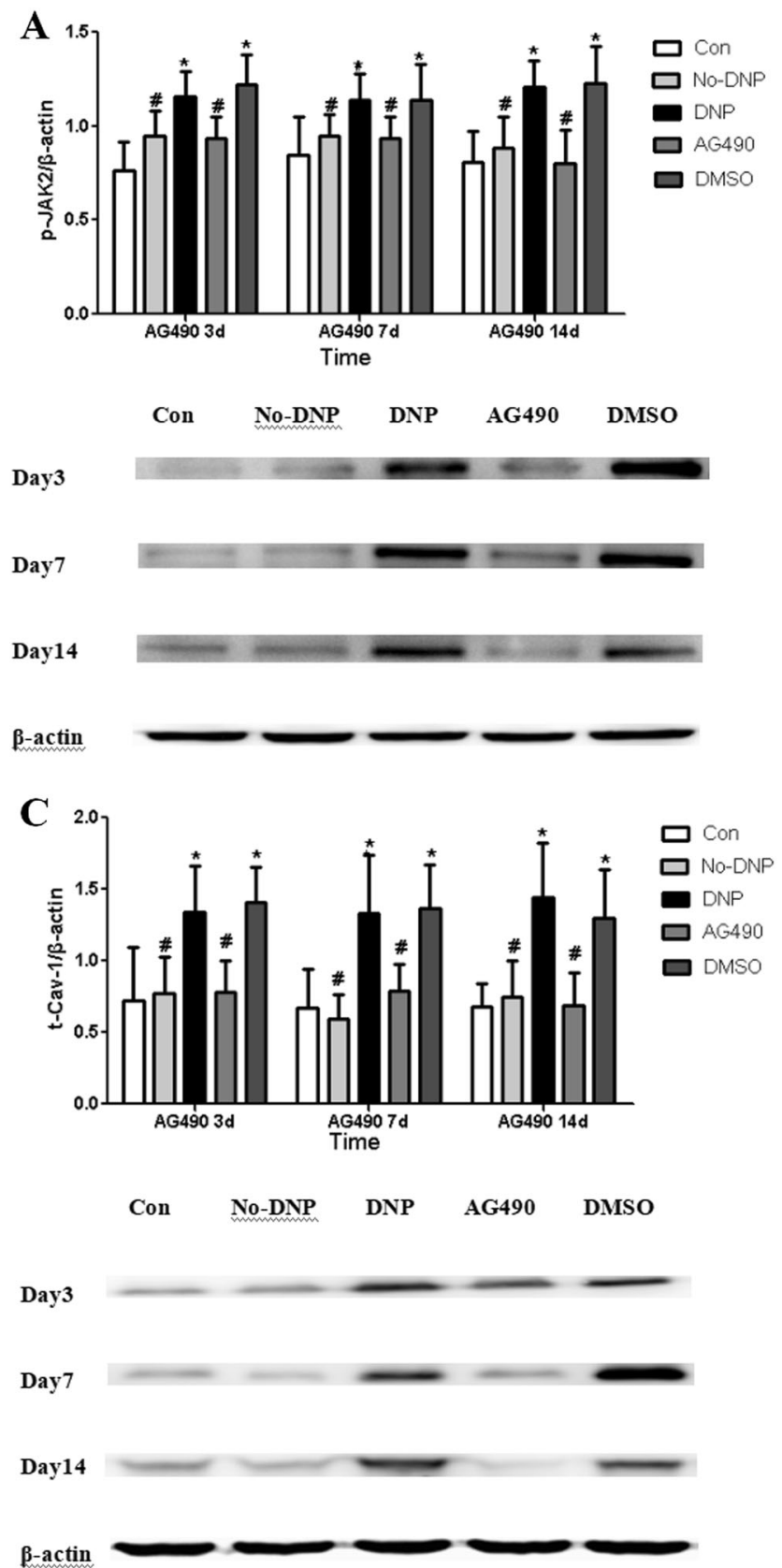

Fig. 5 The representative immunoblotting bands (bottom) and the quantitative data (top) showed following points. (Bottom of A-D): The expression of p-JAK2, p-STAT3, t-Cav-1 and p-NR2B in spinal cord of group Con, No-DNP, DNP, AG490 and DMSO animals by western

effectively reduced allodynia and hyperalgesia [19, 20]. DNP is associated with a slower rate of deafferentation compared with traumatic neuropathy, but, recently, microglia cells also play a role in the development of neuropathic pain in DNP [21]. In the present study, the activation of microglia and astrocytes were both observed in DNP in vivo.

STAT3 mainly expressed in microglia is one of the marker proteins of central nervous system injury. It plays a role in regulating the cytokine-mediated signaling pathway
B
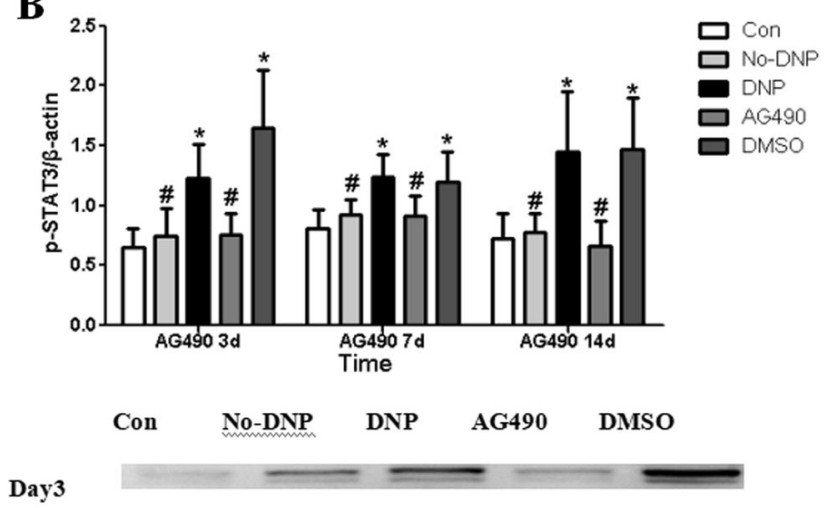

Day 7

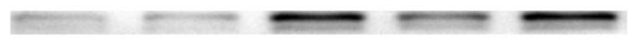

Day14

B-actin
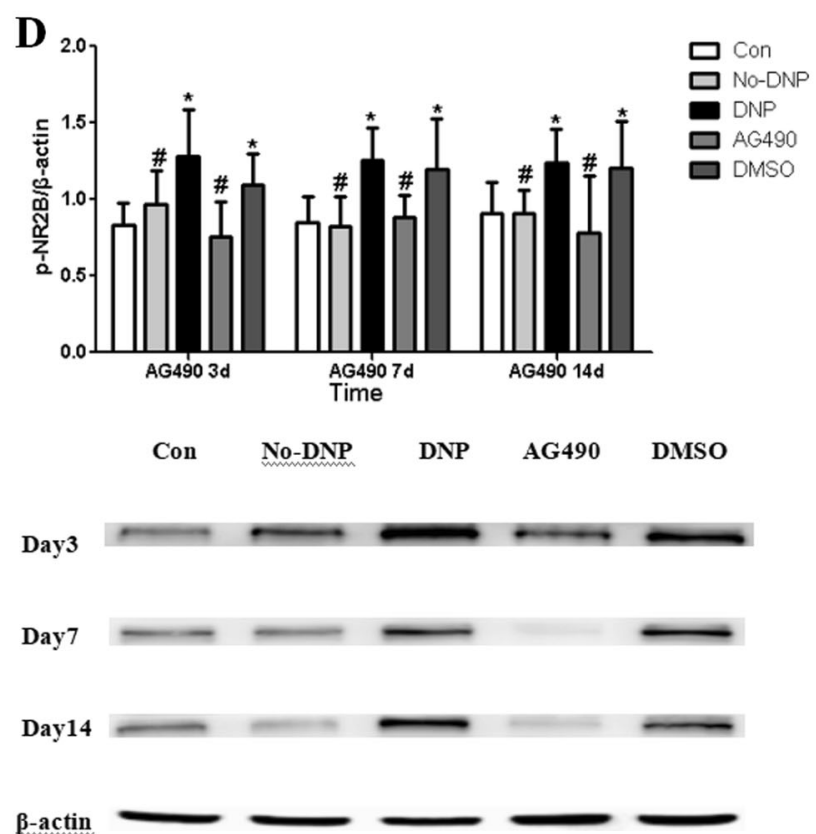

blotting experiment. (Top of A-D): The western blots analysis for the p-JAK2, p-STAT3, t-CAV-1, and p-NR2B protein ( $n=5$ /group) in the different group at different time point. $* P<0.05$ vs. Con group; $\# P<$ 0.05 vs. Painful diabetic neuropathy (DNP) group

[22]. In the context of maladaptive plasticity, abnormal signaling between glia and neurons also has a role in pain. After traumatic nerve injury, microglia in the dorsal horn of the spinal cord release factors such as brain-derived neurotrophic factor (BDNF), leading to the amplification of nociceptive synaptic processing, resulting in "gating" of neuropathic pain [23]. In the CCI model, and in the earlystage of pain, the double-label immunofluorescence assays revealed that p-STAT3 was mainly co-localized with 
A a
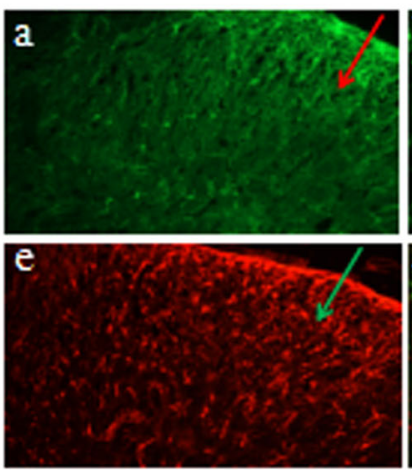

$\mathrm{B}$

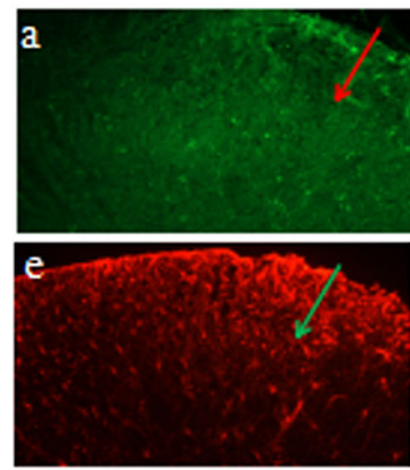

$\mathrm{C}$
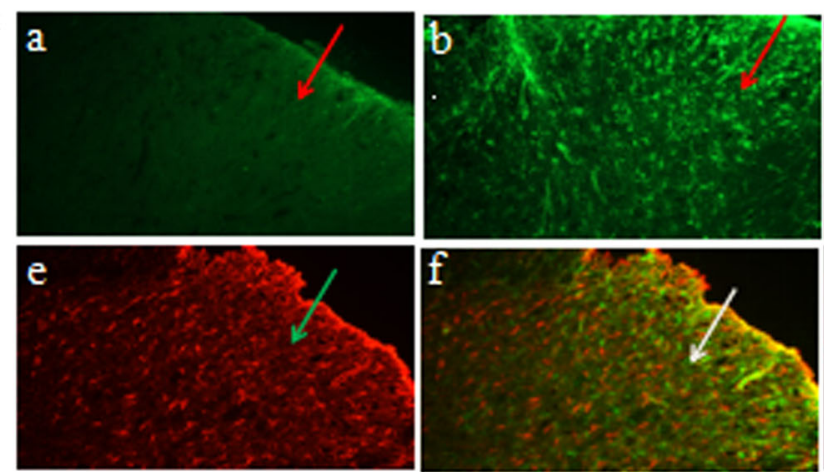

Fig. 6 The expression of p-STAT3-IR and localization of p-STAT3 mainly with microglia, but astrocytes and neuron, in the spinal cord dorsal horn of the type-2 painful diabetic neuropathy (DNP) rats. A The third day after AG490 intrathecal administration. B The seventh day after AG490 intrathecal administration. C The fourteenth day after AG490 intrathecal administration. There are numerous p-STAT3-IR exists in the superficial and medial laminae (I-V) of spinal cord horn at group DNP in picture Aa-b, Ba-b, Ca-b (the arrows to the bright green in the pictures). The microglia cells (OX-42, red arrows highlight) and the co-label of p-STAT3-IR with microglial cells (arrow highlights in

microglia [7]. However, some studies have indicated that STAT3 co-exists with astrocytes, and participates in the activation of astrocytes [13, 24, 25]. In the present study, the double-label immunofluorescence assays revealed that p-STAT3 was mainly expressed in microglia in the spinal cord, which was not consistent with some of the conclusions above. This may be due to the different model of neuropathic pain, such as the particularity of the hyperglycemia state in DNP model. Moreover, it was found that the
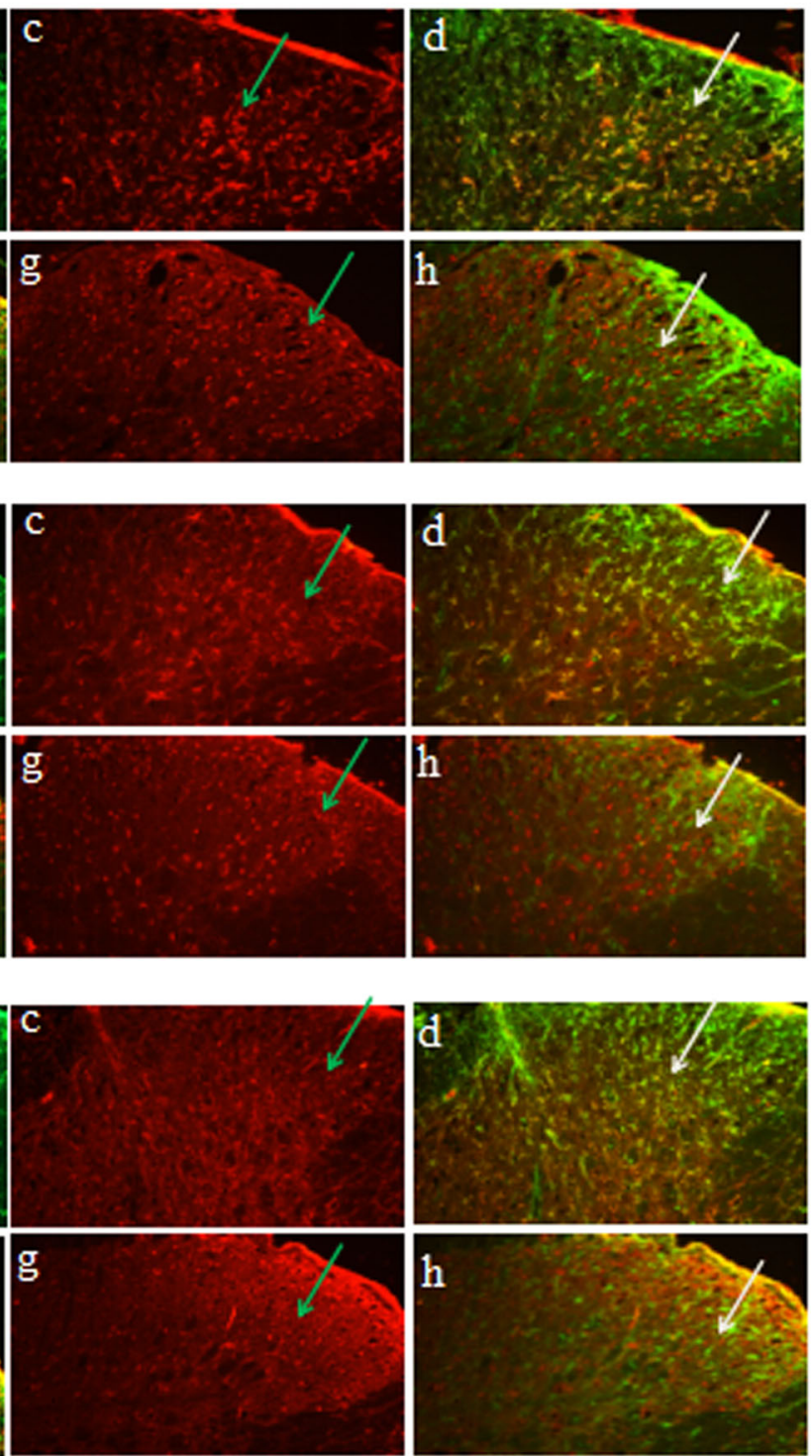

yellow), astrocytes cell markers GFAP (the arrows to the red highlight of GFAP, yellow highlights for the co-label) and neurons markers NeuN (the arrows to the red highlight of NeuN, yellow highlights for co-label) by double immunofluorescence table experiments, respectively (pictures Ac-f, Bc-f, Cc-f). Neither astrocytes nor neurons in spinal cord of DNP animals are co-labeled with p-STAT3-IR at the third, seventh, and fourteenth days. Three independent experiments were performed with different animals from each experimental group for immunohistochemical experiments $(n=6)$. Tissue slices, $20 \mu \mathrm{m}$. Scale bar, $100 \mu \mathrm{m}$

high-glucose environment upregulated the expression of $\mathrm{p}$ STAT3 in microglia, which was consistent with what was found in vitro.

\section{The correlation between CAV-1 and STAT3}

Caveolae is a specialized type of lipid raft stabilized by oligomers of caveolin protein. Caveolae has a large number of membrane-bound proteins, which are mostly signal 


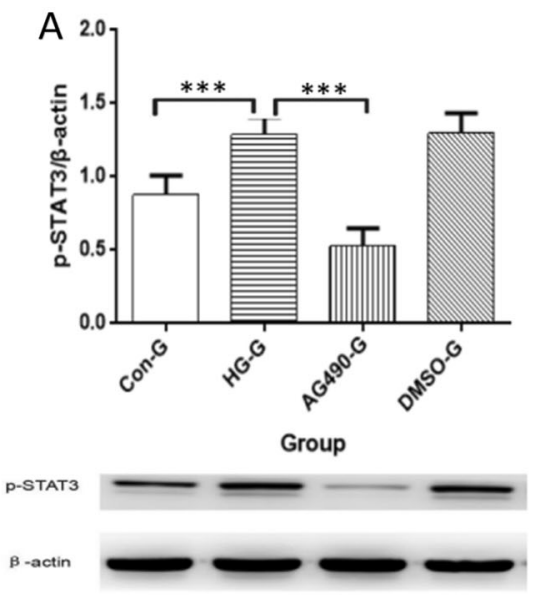

B

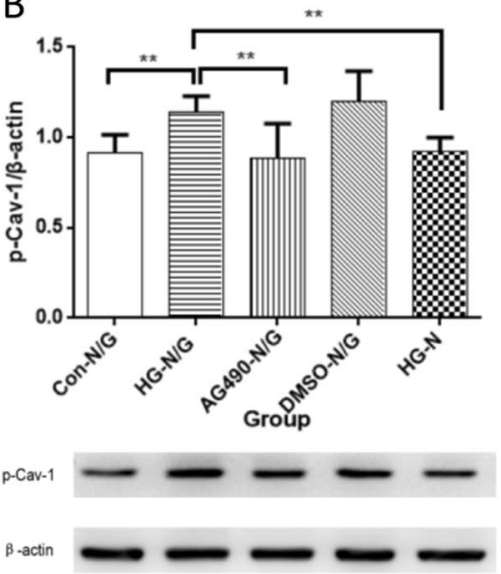

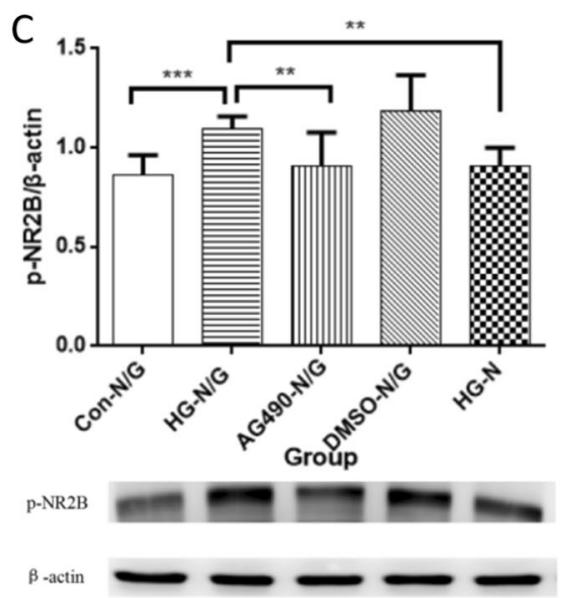

Fig. 7 The results of cell culture in vitro. A The expression of $\mathrm{p}$ STAT3 in BV2 cells under high glucose. Mean \pm SD. $n=3$. $* * * P<$ 0.001 . B The expression of p-CAV-1 in PC12 cells under co-culture system. Mean \pm SD. $n=3$. $* * P<0.01$. C The expression of $\mathrm{p}-\mathrm{NR} 2 \mathrm{~B}$ in PC12 cells under co-culture system. Mean \pm SD. $n=3$. $* * P<0.01$, $* * * P<0.001$ molecules with lipid modification. Caveolae is a platform for the exchange of these signaling molecules, allowing the signal pathways to interact with each other. CAV-1 is the crucial adjustment of the signal pathways in each platform. In general, CAV-1 has a negative regulatory signal transduction pathway for signaling molecules in Caveolae, and it also has the function of enhancing the signal [26-28].

CAV-1 has been shown to directly interact with STAT3 on lipid rafts [29, 30]. Activated STATs leave a lipid raft into the cell, and the subsequent cross-cytoplasm transport requires a specific chaperone protein, in which CAV-1 is one of the chaperone proteins [31]. Research has shown that the JAK/STAT signaling pathway locates on the caveolae containing CAV-1 [32, 33]. CAV-1 is an important gene targeting of STAT3, and STAT3 directly affects the transcription of CAV-1 by directly binding to the CAV-1 promoter. The overexpression of p-STAT3 causes CAV-1 promoter activation and the increase in gene expression [9].

In the present study, in the dorsal horn of the spinal cord, the expression of t-CAV-1 was upregulated in vivo. Owing to laboratory conditions, the expression and distribution of p-CAV-1 in the dorsal horn of the spinal cord were not detected in the present study. In the high-glucose environment, the expression of p-STAT3 was upregulated in BV2, and the expression of $\mathrm{p}-\mathrm{CAV}-1$ was upregulated in the coculture of BV2 and PC12. In contrast, the expression of pCAV-1 was not upregulated in PC12 alone. Hence, it can be speculated that p-CAV-1 may be a downstream target of $\mathrm{p}$ STAT3. In addition, in vivo and in vitro experiments have confirmed that AG490 can relieve the abnormal expression of CAV-1. In view of the exact relationship with the JAK/ STAT signaling pathway, the above-mentioned inference can be further confirmed. However, since the present study did not directly interpret the STAT3-CAV-1 signaling pathway, further experiments were needed to confirm whether CAV-1 acts as an important downstream target of the JAK/STAT pathway in the pathogenesis of DNP.

\section{The correlation between CAV-1 and NR2B}

The central sensitization caused by NMDAR plays an important role in the formation and development of DNP. A number of studies have shown that NR2B activation and neuropathic pain are closely correlated to its production and maintenance [34, 35].

HEAD BP found a close association between CAV-1 and NR2B in the primary culture of rat cortical neurons [31]. CAV-1 regulates neuronal plasticity and receptor transport, regulating NR2B-NMDAR, which is closely correlated to neuropathic pain and central sensitization. In the CCI model, the expression levels of CAV-1 and NR2B in the anterior cingulate cortex were significantly higher than those in normal rats. CAV-1 promoted the expression of NR2B on the membrane, and opened the ion channel, thereby altering neuronal synaptic plasticity, and causing central sensitization. In addition, the increase in NR2B expression can be inhibited by CAV-1 siRNA or NR2B inhibitors. The coimmunoprecipitation and two-hybrid assay also demonstrated a direct interaction between CAV-1 and NR2B. CAV-1 regulated chronic neuropathic pain by modulating NR2B in the anterior cingulate gyrus [10].

In the present study, it was found that the expression of p-NR2B in the dorsal horn of DNP rats was upregulated. In the in vitro experiments, it was also found that the highglucose environment could increase the expression of $\mathrm{p}$ NR2B in neurons. As the present study did not intervene with the CAV-1-NR2B pathway, it was difficult to elucidate the correlation between these two in the pathogenesis of DNP. In addition, the in vivo and in vitro experiments confirmed that AG490 can alleviate the abnormal 
expression of $\mathrm{p}-\mathrm{NR} 2 \mathrm{~B}$, in view of the important position of NMDA in the pathogenesis of neuropathic pain. Furthermore, to a certain extent, it can reflect the significance of the JAK2/STAT3 signaling pathway in DNP treatment.

\section{Influence of high-glucose on the JAK2/STAT3-CAV-1- NR2B signaling pathway}

Persistent high blood glucose level in diabetes plays an initiation role in the change of voltage gated calcium channel and voltage gated sodium channel in the cytomembrane of neuron axons, and the release of neural growth active substances and $\mathrm{P}$ substances [36]. This thereby upregulates the excitability of peripheral sensory nerve fibers and neurons in the spinal dorsal horn, leading to the spontaneous discharge activities of neurons and sensitization to stimulation, which may be the foundation of neuropathic pain. In the present study, it was found that high glucose may have an effect on the JAK2/STAT3CAV-1-NR2B signal pathway, and the cell experiment indicated that it may affect p-CAV-1 and p-NR2B in PC12 cells through the upregulation of p-STAT3 on BV2 cells.

Several observations have confirmed that high glucose has a major role in the upregulation of p-JAK2 and pSTAT3 in mesangial cells [37]. Mao et al. [38] found that the high-glucose-induced JAK/STAT signaling pathway is activated in human glomerular mesangial cells. In addition, under high-glucose conditions, the phosphorylation of CAV-1 was significantly increased in podocytes [39]. Furthermore, CAV-1 expression has been observed in monolayer ECs exposed to high glucose [40]. Moreover, the expression of NR2B is also upregulated in retinal ganglion cells-5 under high-glucose conditions [41]. A positive correlation between poor control of blood glucose and severity of neuropathy or the risk and intensity of neuropathic pain was also detected [42]. Nevertheless, this correlation is not a linear relationship as some patients have severe neuropathy but do not develop neuropathic pain.

In the present in vivo experiments, $56 \%$ of T2DM rats successfully progressed to DNP, while the remaining T2DM rats did not present with neuropathic pain. Compared with the DNP group, the expression levels of p-JAK2, p-STAT3, CAV-1, and p-NR2B in the spinal dorsal horn in the no-DNP group were not significantly different, and this was the same with the Con group. Moreover, there was no difference in blood glucose levels between the DNP group and no-DNP group at all time points, and all remained at a high level. Therefore, in contradiction with the results of the in vitro experiments, the in vivo experiments confirmed that hyperglycemia did not affect the expression of p-JAK2, pSTAT3, CAV-1, and p-NR2B.
The following reasons may contribute to the differences observed in vivo and in vitro: (1) The expression of proteins was detected in certain cells in vitro, while this was detected in all cells in the spinal dorsal horn in vivo. (2) The concentration of glucose in the high-glucose group $(33.3 \mathrm{mM})$ in vitro was pretty approximate to the blood glucose detected in T2DM rats $(30.2 \pm 4.8 \mathrm{mM})$. However, compared with the simple environment in vitro experiment, the results induced by high glucose in vitro may not be consistent with the results under the complex organism environment, involving multiple systems, organs and multiple cells in the in vivo experiment. It was also considered that the high blood glucose level under the complex environment in vivo may have some effects on the expression of some proteins in the JAK2/STAT3-CAV-1-NR2B signal pathway in certain cells of the spinal cord. However, this was not significant, and this pathway may be more correlated to the pathogenesis of neuropathic pain, rather than merely high blood glucose.

In vitro, the high glucose environment can significantly affect the expression of related proteins of the JAK2/ STAT3-CAV-1-NR2B pathway. In vivo, high blood glucose levels have no significant effect on the expression of the protein, but each protein of the DNP rat spinal dorsal horn were abnormally expressed, and the JAK2 specific inhibitors could inhibit the above abnormal expressions in vivo and in vitro. Each protein of the JAK2/STAT3CAV-1-NR2B pathway plays an important role in the pathogenesis of DNP. However, the specific signal transduction between the upstream and downstream of CAV-1 needs to be further confirmed.

Funding This study was supported by National Natural Science Foundation of China (Project No. 81771487) and Zhejiang Provincial Natural Science Foundation of China (Project No. LY17H070006).

\section{Compliance with ethical standards}

Conflict of interest The authors declare that they have no conflict of interest.

Ethical approval The study protocol was approved by the Animal Research Committee of Wenzhou Medical University. All applicable international, national, and/or institutional guidelines for the care and use of animals were followed. This article does not contain any studies with human participants performed by any of the authors.

Publisher's note: Springer Nature remains neutral with regard to jurisdictional claims in published maps and institutional affiliations.

Open Access This article is distributed under the terms of the Creative Commons Attribution 4.0 International License (http://crea tivecommons.org/licenses/by/4.0/), which permits use, duplication, adaptation, distribution, and reproduction in any medium or format, as long as you give appropriate credit to the original author(s) and the 
source, provide a link to the Creative Commons license, and indicate if changes were made.

\section{References}

1. S. Tesfaye, A.J. Boulton, P.J. Dyck, R. Freeman, M. Horowitz, P. Kempler, G. Lauria, R.A. Malik, V. Spallone, A. Vinik, L. Bernardi, P. Valensi; Toronto Diabetic Neuropathy Expert Group, Diabetic neuropathies: update on definitions, diagnostic criteria, estimation of severity, and treatments. Diabetes Care 33, 2285-2293 (2010)

2. M. Tsuda, Microglia in the spinal cord and neuropathic pain. J. Diabetes Investig. 7, 17-26 (2016)

3. J. Molet, A. Mauborgne, M. Diallo, V. Armand, D. Geny, L. Villanueva, Y. Boucher, M. Pohl, Microglial Janus kinase/signal transduction and activator of transcription 3 pathway activity directly impacts astrocyte and spinal neuron characteristics. J. Neurochem. 136, 133-147 (2016)

4. Y. Lei, Y. Sun, C. Lu, Z. Ma, X. Gu, Activated glia increased the level of proinflammatory cytokines in a resiniferatoxin-induced neuropathic pain rat model. Reg. Anesth. Pain. Med. 41, 744-749 (2016)

5. S.R. Chen, G. Samoriski, H.L. Pan, Antinociceptive effects of chronic administration of uncompetitive NMDA receptor antagonists in a rat model of diabetic neuropathic pain. Neuropharmacology 57, 121-126 (2009)

6. R.R. Ji, T. Berta, M. Nedergaard, Glia and pain: is chronic pain a gliopathy? Pain 154(Suppl 1), S10-S28 (2013)

7. E. Dominguez, C. Rivat, B. Pommier, A. Mauborgne, M. Pohl, JAK/STAT3 pathway is activated in spinal cord microglia after peripheral nerve injury and contributes to neuropathic pain development in rat. J. Neurochem. 107, 50-60 (2008)

8. S. Liu, W.L. Mi, Q. Li, M.T. Zhang, P. Han, S. Hu, Q.L. Mao-Ying, Y.Q. Wang, Spinal IL-33/ST2 signaling contributes to neuropathic pain via neuronal CaMKII-CREB and astroglial JAK2-STAT3 cascades in mice. Anesthesiology 123, 1154-1169 (2015)

9. W.T. Chiu, H.T. Lee, F.J. Huang, K.D. Aldape, J. Yao, P.S. Steeg, C.Y. Chou, Z. Lu, K. Xie, S. Huang, Caveolin-1 upregulation mediates suppression of primary breast tumor growth and brain metastases by stat3 inhibition. Cancer Res. 71, 4932-4943 (2011)

10. J.X. Yang, L. Hua, Y.Q. Li, Y.Y. Jiang, D. Han, H. Liu, Q.Q. Tang, X.N. Yang, C. Yin, L.Y. Hao, L. Yu, P. Wu, C.J. Shao, H. L. Ding, Y.M. Zhang, J.L. Cao, Caveolin-1 in the anterior cingulate cortex modulates chronic neuropathic pain via regulation of NMDA receptor 2B subunit. J. Neurosci. 35, 36-52 (2015)

11. J.K. Dang, Y. Wu, H. Cao, B. Meng, C.C. Huang, G. Chen, J. Li, X.J. Song, Q.Q. Lian, Establishment of a rat model of type II diabetic neuropathic pain. Pain Med. 15, 637-646 (2014)

12. B. Meng, L.L. Shen, X.T. Shi, Y.S. Gong, X.F. Fan, J. Li, H. Cao, Effects of curcumin on TTX-R sodium currents of dorsal root ganglion neurons in type 2 diabetic rats with diabetic neuropathic pain. Neurosci. Lett. 605, 59-64 (2015)

13. J.E. Herrmann, T. Imura, B. Song, J. Qi, Y. Ao, T.K. Nguyen, R. A. Korsak, K. Takeda, S. Akira, M.V. Sofroniew, STAT3 is a critical regulator of astrogliosis and scar formation after spinal cord injury. J. Neurosci. 28, 7231-7243 (2008)

14. D. Li, Y. Yan, L. Yu, Y. Duan, Procaine attenuates pain behaviors of neuropathic pain model rats possibly via inhibiting JAK2/ STAT3. Biomol. Ther. (Seoul.) 24, 489-494 (2016)

15. D.I. Orellana, R.A. Quintanilla, C. Gonzalez-Billault, R.B. Maccioni, Role of the JAKs/STATs pathway in the intracellular calcium changes induced by interleukin- 6 in hippocampal neurons. Neurotox. Res. 8, 295-304 (2005)
16. T.J. Mitchell, S. John, Signal transducer and activator of transcription (STAT) signalling and T-cell lymphomas. Immunology 114, 301-312 (2005)

17. P. Calmels, G. Mick, B. Perrouin-Verbe, M. Ventura, SOFMER (French Society for Physical Medicine and Rehabilitation), Neuropathic pain in spinal cord injury: identification, classification, evaluation. Ann. Phys. Rehabil. Med. 52, 83-102 (2009)

18. A.K. Clark, D. Gruber-Schoffnegger, R. Drdla-Schutting, K.J. Gerhold, M. Malcangio, J. Sandkühler, Selective activation of microglia facilitates synaptic strength. J. Neurosci. 35, 4552-4570 (2015)

19. Y. Liang, Y. Qiu, J. Du, J. Liu, J. Fang, J. Zhu, J. Fang, Inhibition of spinal microglia and astrocytes contributes to the anti-allodynic effect of electroacupuncture in neuropathic pain induced by spinal nerve ligation. Acupunct. Med. 34, 40-47 (2016)

20. Y. Yang, H. Li, T.T. Li, H. Luo, X.Y. Gu, N. Lü, R.R. Ji, Y.Q. Zhang, Delayed activation of spinal microglia contributes to the maintenance of bone cancer pain in female Wistar rats via P2X7 receptor and IL-18. J. Neurosci. 35, 7950-7963 (2015)

21. M. Tsuda, H. Ueno, A. Kataoka, H. Tozaki-Saitoh, K. Inoue, Activation of dorsal horn microglia contributes to diabetesinduced tactile allodynia via extracellular signal-regulated protein kinase signaling. Glia 56, 378-386 (2008)

22. X. Yang, G. He, Y. Hao, C. Chen, M. Li, Y. Wang, G. Zhang, Z. $\mathrm{Yu}$, The role of the JAK2-STAT3 pathway in pro-inflammatory responses of EMF-stimulated N9 microglial cells. J. Neuroinflamm. 7, 54 (2010)

23. S. Beggs, T. Trang, M.W. Salter, P2X4R+microglia drive neuropathic pain. Nat. Neurosci. 15, 1068-1073 (2012)

24. M. Tsuda, Y. Kohro, T. Yano, T. Tsujikawa, J. Kitano, H. TozakiSaitoh, S. Koyanagi, S. Ohdo, R.R. Ji, M.W. Salter, K. Inoue, JAK-STAT3 pathway regulates spinal astrocyte proliferation and neuropathic pain maintenance in rats. Brain 134, 1127-1139 (2011)

25. D.S. Aaronson, C.M. Horvath, A road map for those who don't know JAK-STAT. Science 296, 1653-1655 (2002)

26. B. Razani, J.A. Engelman, X.B. Wang, W. Schubert, X.L. Zhang, C.B. Marks, F. Macaluso, R.G. Russell, M. Li, R.G. Pestell, D. Di Vizio, H. Hou Jr, B. Kneitz, G. Lagaud, G.J. Christ, W. Edelmann, M.P. Lisanti, Caveolin-1 null mice are viable but show evidence of hyperproliferative and vascular abnormalities. J. Biol. Chem. 276, 38121-38138 (2001)

27. K. Abdelmohsen, Y. Kuwano, H.H. Kim, M. Gorospe, Posttranscriptional gene regulation by RNA-binding proteins during oxidative stress: implications for cellular senescence. Biol. Chem. 389, 243-255 (2008)

28. Y. Jin, S.J. Lee, R.D. Minshall, A.M. Choi, Caveolin-1: a critical regulator of lung injury. Am. J. Physiol. Lung Cell. Mol. Physiol. 300, L151-L160 (2011)

29. P.B. Sehgal, G.G. Guo, M. Shah, V. Kumar, K. Patel, Cytokine signaling: STATS in plasma membrane rafts. J. Biol. Chem. 277, 12067-12074 (2002)

30. G. Shen-Tu, D.B. Schauer, N.L. Jones, P.M. Sherman, Detergentresistant microdomains mediate activation of host cell signaling in response to attaching-effacing bacteria. Lab. Invest. 90, 266-281 (2009)

31. B.P. Head, H.H. Patel, Y.M. Tsutsumi, Y. Hu, T. Mejia, R.C. Mora, P.A. Insel, D.M. Roth, J.C. Drummond, P.M. Patel, Caveolin-1 expression is essential for N-methyl-D-aspartate receptor-mediated $\mathrm{Src}$ and extracellular signal-regulated kinase $1 / 2$ activation and protection of primary neurons from ischemic cell death. FASEB J. 22, 828-840 (2008)

32. N. Ariotti, R.G. Parton, SnapShot: caveolae, caveolins, and cavins. Cell 154, 704-704.e1 (2013)

33. Y. Liu, Z. Liang, J. Liu, W. Zou, X. Li, Y. Wang, L. An, Downregulation of caveolin-1 contributes to the synaptic 
plasticity deficit in the hippocampus of aged rats. Neural Regen. Res. 8, 2725-2733 (2013)

34. W. Zhang, C.X. Shi, X.P. Gu, Z.L. Ma, W. Zhu, Ifenprodil induced antinociception and decreased the expression of NR2B subunits in the dorsal horn after chronic dorsal root ganglia compression in rats. Anesth. Analg. 108, 1015-1020 (2009)

35. M. Zhuo, Plasticity of NMDA receptor NR2B subunit in memory and chronic pain. Mol. Brain 2, 4 (2009)

36. L. Manni, F. Florenzano, L. Aloe, Electroacupuncture counteracts the development of thermal hyperalgesia and the alteration of nerve growth factor and sensory neuromodulators induced by streptozotocin in adult rats. Diabetologia 54, 1900-1908 (2011)

37. Y.H. Shi, S. Zhao, C. Wang, Y. Li, H.J. Duan, Fluvastatin inhibits activation of JAK and STAT proteins in diabetic rat glomeruli and mesangial cells under high glucose conditions. Acta Pharmacol. Sin. 28, 1938-1946 (2007)

38. T. Mao, H. Chen, L. Hong, J. Li, Pigment epithelium-derived factor inhibits high glucose-induced JAK/STAT signalling pathway activation in human glomerular mesangial cells. Saudi. Med. J. 34, 793-800 (2013)

39. L.N. Sun, Z.X. Chen, X.C. Liu, H.Y. Liu, G.J. Guan, G. Liu, Curcumin ameliorates epithelial-to-mesenchymal transition of podocytes in vivo and in vitro via regulating caveolin-1. Biomed. Pharmacother. 68, 1079-1088 (2014)

40. C. Tian, R. Zhang, X. Ye, C. Zhang, X. Jin, Y. Yamori, L. Hao, $X$. Sun, C. Ying, Resveratrol ameliorates high-glucose-induced hyperpermeability mediated by caveolae via VEGF/KDR pathway. Genes Nutr. 8, 231-239 (2013)

41. W. Qu, B. Zhang, Z.F. Zuo, X.Z. Liu, [The role of NR2B in the apoptosis of retinal ganglion cells-5 induced by glucose]. Chin. J. Anat. 38, 576-578 (2015). [Article in Chinese]

42. A.C. Themistocleous, J.D. Ramirez, P.R. Shillo, J.G. Lees, D. Selvarajah, C. Orengo, S. Tesfaye, A.S. Rice, D.L. Bennett, The Pain in Neuropathy Study (PiNS): a cross-sectional observational study determining the somatosensory phenotype of painful and painless diabetic neuropathy. Pain 157, 1132-1145 (2016) 